\title{
Role of primary miRNA polymorphic variants in metastatic colon cancer patients treated with 5-fluorouracil and irinotecan
}

\author{
V Boni ${ }^{1}$, R Zarate ${ }^{2}$, JC Villa ${ }^{3}$, \\ E Bandrés ${ }^{2}$, MA Gomez ${ }^{3}$, \\ E Maiello $^{1}$, J Garcia-Foncillas ${ }^{2}$ \\ and $\mathrm{E}$ Aranda ${ }^{3}$

\begin{abstract}
${ }^{1}$ Oncology Unit, Department of Oncology and Haematology, Casa Sollievo Sofferenza,

S. Giovanni Rotondo, Italy; ${ }^{2}$ Laboratory of Pharmacogenomics, Department of Oncology, Center for Applied Medical Research, University of Navarra, Pamplona, Spain and ${ }^{3}$ Medical Oncology Service, Hospital Universitario Reina Sofía, Córdoba, Spain
\end{abstract}

\section{Correspondence:}

Dr J Garcia-Foncillas, Laboratory of Pharmacogenomics, Center for Applied Medical Research, University of Navarra, Avda Pio XII 55, 31008 Pamplona, Spain. E-mail: jgfoncillas@unav.es and Dr E Aranda, Medical Oncology Service, Hospital Universitario Reina Sofía, Avda Menéndez Pidal s.n., 14004 Córdoba, Spain.

E-mail: earandaa@seom.org
Received 16 December 2009; revised 19 March 2010; accepted 4 May 2010; published online 29 June 2010
MicroRNAs are non-coding RNAs that can block mRNA translation and influence mRNA stability. Recent evidence indicates that miRNA variations can affect drug resistance, efficacy, and metabolism, opening new avenues of pharmacogenomics research. We investigated associations between polymorphisms in both miRNA-containing genomic regions (primary and precursor miRNA) and in genes related to miRNA biogenesis with clinical outcome in metastatic colorectal cancer (mCRC) patients treated with 5-fluorouracil and irinotecan (CPT-11). Eighteen single-nucleotide polymorphisms (SNPs) were analyzed in 61 patients. A significant association with tumor response and time to progression (TTP) was found for SNP rs7372209 in pri-miR26a-1 $(P=0.041$ and $P=0.017$, respectively). The genotypes CC and CT were favorable when compared with the $\Pi T$ variant genotype. In addition, SNP rs1834306, located in the pri-miR-100 gene, significantly correlated with a longer TTP $(P=0.04)$. In the miRNA-biogenesis pathway, a trend was identified between SNP rs 11077 in the exportin-5 gene and disease control rate $(P=0.076)$. This study is the first to suggest a relationship between treatment outcome and SNPs in the miRNA-biogenesis machinery, in both primary and precursor miRNAs. Our results suggest that miRNA polymorphic variants might be useful predictors of clinical outcome in $\mathrm{mCRC}$ patients treated with 5-fluorouracil and CPT-11 combination.

The Pharmacogenomics Journal (2011) 11, 429-436; doi:10.1038/tpj.2010.58; published online 29 June 2010

Keywords: microRNA; single-nucleotide polymorphism; tumor response; colorectal cancer; 5-FU; irinotecan

\section{Introduction}

Colorectal cancer is the third most common cancer and the second leading cause of cancer death in the Western world. ${ }^{1}$ Five-year mortality rates have modestly declined over the last three decades; however, the need remains for new predictive and prognostic biomarkers that can improve the ability of clinicians to determine the most effective treatment. This approach would allow to identify populations most likely to benefit from a specific type of therapy and a necessary step toward a tailored-made therapy. Pharmacogenomic studies emphasize on the use of genomic information to enhance the success of a given therapy, but recent evidence also indicates that mechanisms involving regulation of gene expression influence drug response. 
MicroRNAs are non-coding, evolutionarily conserved RNAs that, through the regulation of gene expression at the post-transcriptional level, have an important role in multiple cellular processes. Several findings suggest that miRNAs affect chemosensitivity and drug resistance in cancer. $^{2-4}$ Meng et al. ${ }^{2}$ found that miR-21 and miR-200b inhibition increased the sensitivity of cholangiocarcinoma cell lines to gemcitabine by phosphatase and tensin homolog gene regulation. In addition, our group reported that the overexpression of miR-451 increases radiosensitivity in gastric cancer cells. ${ }^{3}$ More recently, it was shown, that at the translational level, miR-192 regulates the dihydrofolate reductase gene, an important target for anticancer drugs such as methotrexate, with the subsequent increase in methotrexate cell sensitivity in vitro. ${ }^{4}$

In addition, recent studies have reported that sequence variations in miRNA regions and miRNA-processing pathways may affect expression levels of mature miRNAs., Although single-nucleotide polymorphisms (SNPs) in miRNA regions are rare and considered unlikely to be functionally important, ${ }^{7}$ nucleotide variations within the seed sequence of the miRNA or within the sequence on primary (pri) and precursor (pre) miRNAs might affect miRNA processing and ultimately lead to modification of miRNA expression. ${ }^{8}$ Therefore, it is plausible that SNPs in miRNAbiogenesis machinery genes and miRNA-containing genomic regions may have a role in cancer development and treatment response. In this sense, the concept of miRNAs SNPs as a source of genetic variability that can influence the way that patients activate and metabolize drugs is emerging. ${ }^{9,10}$ The pharmacogenomic analysis of miRNAs may represent a novel and promising field of research that holds new possibilities for predicting drug behavior and improving drug efficiency.

Altered miRNA expression also has been strongly linked to cancer. ${ }^{11-14}$ Several factors have been implicated in miRNA abnormal expression in tumors, including gene amplification, deletion, epigenetic alterations, and abnormalities in miRNA processing and single-nucleotide substitution resulting in a mutation or SNP. ${ }^{15-17}$ An miRNA is a 22 nucleotides (nt) in length RNA that is transcribed as a pri-miRNA by RNA polymerase II. ${ }^{18}$ The pri-miRNA is cropped to release a hairpin-shaped precursor (pre-miRNA) of about $65 \mathrm{nt}$ in length. Drosha (an RNAse III) and its cofactor, DGCR8 (known as Pasha in Drosophila melanogaster), have an essential role in pri-miRNA processing. ${ }^{19,20}$ After this initial step, the nuclear transport factor exportin-5 (XPO5) exports the resulting pre-miRNAs into the cytoplasm by a Ran (a ras-related nuclear protein) GTP-dependent mechanism. ${ }^{21}$ In the cytoplasm, Dicer, a RNase III type protein associated with the transactivation response element RNAbinding protein (TRBP), ${ }^{22}$ recognizes the double-stranded (ds)RNA stem and releases a 21-bp dsRNA, from which the RNA-induced silencing complex will select one strand as the mature miRNA. ${ }^{23}$ This complex also includes GEMIN3 and GEMIN4 and contributes to both miRNA processing and target gene silencing. ${ }^{24,25}$ Finally, the mature miRNAs guide RNA-induced silencing complex to mRNAs that contain the miRNA target sequences ${ }^{26}$ and negatively affect the expression levels of these mRNAs. ${ }^{27}$

Several potential pathways could be under SNP influence. SNPs in miRNA-biogenesis genes might affect the expression of mature miRNAs and consequently miRNA-mediated regulation within the cell. On the other hand, SNPs in miRNA-containing genomic regions might affect the biogenesis of mature miRNAs and thus their expression within the cell. Furthermore, because small variations at the level of miRNAs could affect thousands of target mRNAs, SNPs in miRNA-related genes may significantly influence the proteome diversity of cells. Finally, SNPs in miRNA might represent ideal candidate biomarkers for cancer prognosis and prediction of clinical outcome. Thus, it would be of great interest to explore the association of polymorphic variations in miRNA-related genes and cancer therapies efficacy.

To investigate whether polymorphisms in miRNAbiogenesis genes and in miRNA-containing genomic regions may influence the outcome of metastatic colorectal cancer (mCRC) patients treated with 5-fluorouracil (5-FU) and irinotecan, we analyzed DNA obtained from 61 patients with mCRC treated with this combination. We focused on 18 SNPs, 11 of them in genes related to miRNA biogenesis (Drosha, DGCR8, XPO5, Ran, GEMIN3, GEMIN4, and TRBP) and the remaining from precursors (miR-146-a, miR-196a-2, miR-423, and miR-604) and primary miRNAs (miR-26a-1, miR-100, and miR-219-1). We hypothesized that these SNPs may affect miRNA expression, modify the proteome network, and have an impact on patient's clinical outcome.

\section{Materials and methods}

\section{Study population, treatments, and clinical variables}

Sixty-one Caucasian patients were retrospectively recruited from the Medical Oncology Service of the University Hospital Reina Sofía, Córdoba (Spain). Table 1a shows the clinical characteristics of the patient population at the time of their diagnosis. When we began the study (baseline), all patients had unresectable mCRC. Table $1 \mathrm{~b}$ summarizes the characteristics of the patient population at baseline. They received a first-line chemotherapy with 5 -FU $\left(2250 \mathrm{mg} \mathrm{m}^{-2}\right.$ as a 48-h continuous infusion) and irinotecan (CPT-11, $180 \mathrm{mg} \mathrm{m}^{-2}$ ) on day 1 , every 14 days. Tumor response was evaluated every 12 weeks using computed tomography scans. Response was defined according to Response Evaluation Criteria in Solid Tumors criteria as complete response (CR), partial response (PR), stable disease (SD), or progressive disease (PD). ${ }^{28}$ An evaluation of disease status was conducted. In the absence of disease progression, either clinical or radiological, therapy was continued until unacceptable toxicity or disease progression. At baseline, all patients showed a performance status (Eastern Cooperative Oncology Group) of $0-1$. Thirteen patients (21\%) had previously undergone surgical resection of the metastasis, but at baseline, all patients involved in the study had metastatic and unresectable disease that was at least C1 stage using the 
Table 1 Characteristics of the study population

$\begin{array}{ccccc} & & \text { ORR } & \text { TTP } & \text { OS } \\ \text { n } & \% & \text { P-value* }^{\text {P-value }} & \text { P-value }\end{array}$

(a) Clinico-pathological characteristics at the time of diagnosis Age, years

\begin{tabular}{|c|c|c|c|c|c|}
\hline Median & 63 & & 0.385 & 0.143 & 0.219 \\
\hline Range & $41-77$ & & 0.658 & 0.576 & 0.354 \\
\hline$\leqslant 65$ & 31 & 51 & & & \\
\hline$>65$ & 30 & 49 & & & \\
\hline Sex & & & 0.793 & 0.163 & 0.578 \\
\hline Male & 45 & 74 & & & \\
\hline Female & 16 & 26 & & & \\
\hline Primary tumor site & & & 0.878 & 0.866 & 0.684 \\
\hline Colon & 32 & 52 & & & \\
\hline Rectum & 29 & 48 & & & \\
\hline Differentiation grade & & & 0.551 & 0.19 & 0.586 \\
\hline G1 & 8 & 14 & & & \\
\hline G2 & 43 & 70 & & & \\
\hline G3 & 5 & 8 & & & \\
\hline$G x$ & 5 & 8 & & & \\
\hline Tumoral size & & & 0.638 & 0.882 & 0.988 \\
\hline $\mathrm{T} 2$ & 3 & 5 & & & \\
\hline T3 & 37 & 61 & & & \\
\hline T4 & 17 & 28 & & & \\
\hline Tx & 4 & 6 & & & \\
\hline Ganglion affectation & & & 0.04 & 0.01 & 0.095 \\
\hline No & 11 & 18 & & & \\
\hline N1 & 24 & 39 & & & \\
\hline N2 & 7 & 12 & & & \\
\hline $\mathrm{Nx}$ & 19 & 31 & & & \\
\hline Stage on diagnosis & & & 0.06 & 0.482 & 0.799 \\
\hline II & 14 & 23 & & & \\
\hline III & 20 & 33 & & & \\
\hline IV & 27 & 44 & & & \\
\hline
\end{tabular}

(b) Clinical characteristics at baseline

Metastatic sites

$\begin{array}{lrrlll}\text { Liver } & 36 & 59 & 0.058 & 0.6 & 0.566 \\ \text { Lung } & 20 & 33 & 0.508 & 0.948 & 0.959 \\ \text { Liver and lung } & 6 & 10 & 0.23 & 0.498 & 0.459 \\ \text { Other } & 19 & 31 & 0.009 & 0.851 & 0.459 \\ \text { Organs with metastasis } & & & 0.138 & 0.644 & 0.221 \\ 1 & 44 & 72 & & & \\ \geqslant 2 & 17 & 28 & & & \end{array}$

$\begin{array}{lrrlll}\text { Prior therapy } & & & & & \\ \quad \text { Tumor resection } & 57 & 76 & 0.5105 & 0.8847 & 0.0143 \\ \text { Metastasis resection } & 13 & 21 & 0.149 & 0.998 & 0.399 \\ \text { Adjuvant } & 31 & 51 & 0.004 & 0.159 & 0.877 \\ \text { chemotherapy } & & & & & \\ \text { Radiotherapy } & 11 & 18 & 0.892 & 0.659 & 0.664 \\ \text { Neoadjuvant } & 2 & 3 & - & - & -\end{array}$

Living/deceased

$35 / 26$

Abbreviations: ORR, overall response rate; OS, overall survival; TTP, time to progression.

${ }^{*} P$-values were calculated as Pearson $\chi^{2}$ or Mantel-Haenszel tests for categorical variables and Mann-Whitney or Kruskal-Wallis tests for continuous ones.

${ }^{\dagger} P$-value log-rank test. second grid stage system. ${ }^{29}$ Twenty-eight patients (46\%) who had localized disease (stages II and III) at the time of their diagnosis and two patients (3\%) who had undergone neoadjuvant chemotherapy before metastasis resection had previously received 5-FU, but none had previously been treated with CPT- 11.

This study was approved by the institutional review board, and all patients provided written informed consent before recruitment.

\section{Selection of genes and polymorphisms}

We selected 11 SNPs in miRNA-biogenesis genes on the basis of the following criteria: (1) the SNP should reside in a functional region including exons, promoters, or untranslated regions (UTRs); (2) SNPs should have a reported minor allelic frequency $>0.20$ in a Caucasian population, as recorded in the SNP database (dbSNP: available to the public online at: http://www.ncbi.nlm.nih.gov/SNP). ${ }^{30}$ In the case of multiple potentially functional SNPs within the same haplotype block (defined by the linkage coefficient $r^{2}>0.8$ ), only one SNP that fulfilled our criteria, was included.

To identify SNPs located in pri- and pre-miRNA genes, its precursor sequence was obtained from the microRNA registry (http://microrna.sanger.ac.uk/) and the corresponding genome regions for each miRNA gene by Blast analysis (http://www.ncbi.nlm.nih.gov/genome/seq/HsBlast.htm). The genomic region corresponding to each precursor miRNA was considered, including at least 100 base pair at the $5^{\prime}$ and $3^{\prime}$ ends, which covers the primary precursor of miRNA. Finally, the SNPs were found searching in the SNP database and selected when the minor allelic frequency was $>0.20$. The reported polymorphisms have been previously addressed in two case-control studies in renal ${ }^{31}$ and bladder cancer ${ }^{32}$, but at the time of writing, no data were available regarding the role of SNPs from microRNA-biogenesis genes and SNPs in miRNA-containing genomic regions in relation to treatment outcome. Table 2 summarizes the genes, nucleotide substitutions, position, amino acid changes, reference SNP identification numbers, and observed allele frequencies. Eleven SNPs were in seven genes of miRNA-biogenesis pathway (Drosha, DGCR8, XPO5, Ran, GEMIN3, GEMIN4, and TRBP), four SNPs were in pre-miRNAs (miR-146-a, miR-196a-2, miR-423, and miR-604), and three SNPs were in pri-miRNAs (miR-26a-1, miR-100, and miR-219-1).

\section{Genotyping}

Genomic DNA was isolated from peripheral blood using a QIAamp DNA extraction kit (Qiagen, Barcelona, Spain) according to the manufacturer's protocol. Genotyping was performed using TaqMan SNP Genotyping Assays (Applied Biosystems, Madrid, Spain) (Table 2). Allelic discrimination was carried out using the Applied Biosystems 7300 RealTime PCR System. Duplicate samples were genotyped as a quality control.

\section{Statistical analysis}

Patient characteristics were reported as frequencies and percentages or as a median and interquartile range when 
Table 2 SNPs examined and genotype frequencies

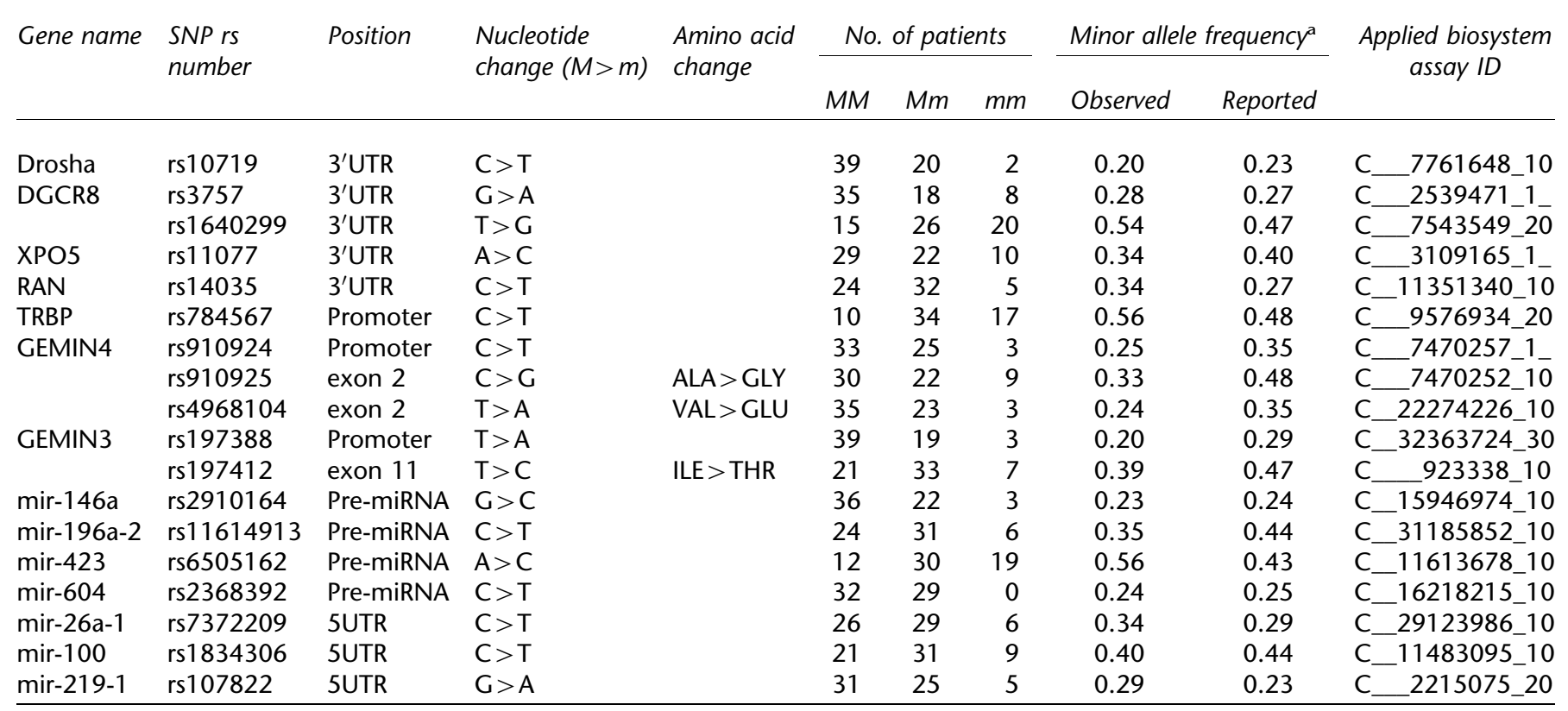

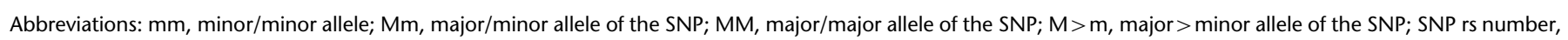
single-nucleotide polymorphism reference identification number.

${ }^{a}$ The reported minor allele frequency was obtained from the dbSNP database.

appropriate. Groups were compared using the Pearson $\chi^{2}$ or Mantel-Haenszel tests for categorical variables and MannWhitney or Kruskal-Wallis tests for continuous variables. The goodness-of-fit $\chi^{2}$ test was adopted to assess the HardyWeinberg equilibrium.

Overall response rate (ORR) was determined by comparing patients showing CR or PR with patients showing SD or PD. In addition to response rate, the disease control rate (DCR), defined as an objective response (CR plus PR) plus SD, was determined and compared with patients showing disease progression. Response to therapy probability was modeled using univariate and multivariate logistic regression. $P$-values refer to the statistical significance of the coefficients. The same approach was followed to model the disease control probability, determined by comparing patients showing CR or PR or SD with patients experiencing PD.

Time to progression (TTP) was calculated from the date of first-line chemotherapy start to the date of first documented progression. Overall survival (OS) was calculated from the date of metastatic colon cancer diagnosis to the date of death. Probabilities of TTP and OS were calculated using the Kaplan-Meier method. Separate progression-free curves were estimated with Kaplan-Meier for SNP genotypes, and the log-rank test was used to assess statistically significant differences among curves. Hazard ratios with their 95\% confidence intervals (95\% CIs) were estimated using the univariate and multivariate Cox proportional hazards model in both TTP and OS analyses.

In the multivariate analysis, the association of each SNP with ORR, DCR, TTP, and OS was tested, and the model was adjusted for possible confounders as well as for SNP interactions. In univariate analyses, $P$-values of all the 18 SNPs examined were corrected for multiple comparisons using permutation resampling techniques. ${ }^{33}$ The $P$-values shown in the paper as $P^{*}$ are the corrected ones.

Dominant, recessive, and additive genetic models were considered and tested for each SNP. All analyses were performed using SAS release 9.1.

\section{Results}

\section{SNP frequencies}

As shown in Table 2, genotype frequencies in the patient population were not significantly different from the minor allelic frequency values reported in the SNP database. All of the SNPs have minor allelic frequency values between 0.20 and 0.56 . With the exception of two SNPs, rs3757 and rs2368392, we identified no evidence of departure from Hardy-Weinberg equilibrium.

\section{Association of genotypes with tumor response}

On an intent-to-treat analysis, the ORR was $41 \%$, with 8 (13\%) CR and 17 (28\%) PR. In addition, 29 patients (48\%) achieved SD, whereas PD were documented in 7 patients (11\%). We observed a response rate (CR or PR) in $41 \%$ of the treated patients.

By univariate analysis, lymph node status and adjuvant chemotherapy were significantly associated with ORR. At baseline, we found a significant association of ORR with other metastatic sites such as bone or brain. 
Table 3 Relevant associations of SNP rs7372209 in pri-miR-26a-1 with clinical outcome

\begin{tabular}{|c|c|c|c|c|c|c|c|c|c|c|c|c|}
\hline \multirow[t]{2}{*}{ Genotype } & \multicolumn{6}{|c|}{ ORR } & \multicolumn{6}{|c|}{ TTP } \\
\hline & $\begin{array}{l}C R / P R \\
n(\%)\end{array}$ & $\begin{array}{c}S D / P D \\
n(\%)\end{array}$ & P-value* & $O R^{a}$ & $95 \% \mathrm{Cl}$ & P-value & MST (months) & $95 \% \mathrm{Cl}$ & P-value* & $H R^{\mathrm{b}}$ & $95 \% \mathrm{Cl}$ & $\mathrm{P}$-value ${ }^{\mathrm{b}}$ \\
\hline \multicolumn{13}{|c|}{ miR-26a-1 rs7372209 } \\
\hline $\mathrm{CC} / \mathrm{CT}$ & $23(37)$ & $32(54)$ & & 1 & & & 18.32 & 13.37-24.09 & & 1 & & \\
\hline$\pi$ & $2(3)$ & $4(6)$ & 0.034 & 0.1 & $0.01-0.99$ & 0.044 & 9.18 & $3.96-18.52$ & $0.017^{* *}$ & 2.44 & $1.29-4.61$ & 0.0035 \\
\hline
\end{tabular}

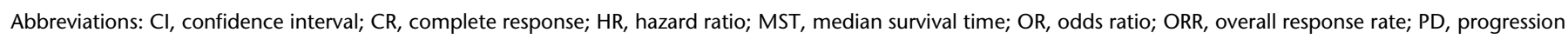
disease; $P R$, partial response; SD, stable disease; TTP, time to progression.

${ }^{a} \mathrm{OR}$ was adjusted for clinical factors. P-value from logistic regression model.

bHR was adjusted for clinical factors. $P$-value from Cox regression model.

${ }^{*} P$-values were adjusted by multiple comparisons with permutation test.

${ }^{* *} P$-value log-rank test.

By univariate analysis, rs7372209 SNP, located in the $5^{\prime}$ region of the pri-miR-26a-1 gene, was significantly associated with ORR $\left(P^{\star}=0.041\right)$. The effect of this SNP on response rate was stronger when homozygote wild type (CC) combined with heterozygote (CT) was compared with variant homozygote $(\mathrm{TT})\left(P^{\star}=0.034\right)$. In multivariate analysis, performed by adjusting for clinical factors, this association remained statistically significant $(P=0.044)$ (Table 3). A trend toward an association with DCR was found for SNP rs11077 in the XPO5 gene when AA and AC genotypes were compared with the polymorphic variant CC $\left(P^{\star}=0.076\right.$ and $P=0.084$ in univariate and multivariate logistic analyses, respectively) (Supplementary Table 1).

\section{Association of genotypes with TTP}

At the time of the final analysis, the median follow-up time was 22.2 months (range, 17.31-31.18 months). A significant association was found between rs7372209 SNP in pri-miR26a-1 and TTP $(P=0.01$ log-rank test). Homozygote wild type (CC) and heterozygote (CT) showed twofold longer median TTP when compared with the homozygote variant (TT) (Figure 1). Patients carrying a C-containing genotype in miR-26a-1 SNP had a median TTP of 18.32 months (95\% CI, 13.37-24.09), whereas median TTP was 9.18 months $(95 \%$ CI, 3.96-18.52) in patients with polymorphic genotype TT variant, in both univariate $\left(P^{*}=0.0017\right)$ and multivariate $(P=0.0035)$ Cox proportional hazards regression analysis (Table 3).

Among the other SNPs analyzed, an association was observed between TTP and SNP rs1834306 located in the $5^{\prime}$ UTR position of the pri-miR-100 gene $\left(P^{\star}=0.04\right.$ log-rank test), although the adjusted Cox analysis showed only a trend toward an association $(P=0.056)$ (Supplementary Table 2). Median TTP for patients with the CC and CT genotypes was 18.52 months (95\% CI, 13.37-23.47) compared with 12.62 months (95\% CI, 7.70-16.75) for patients with the TT polymorphic genotype (Figure 2).

No further associations were found for the remaining miRNA-related genes. We also found a lack of interactions of the unfavorable genotypes of the two SNPs (in miR-26a-1 and miR-100 genes). In addition, none of the SNPs showed

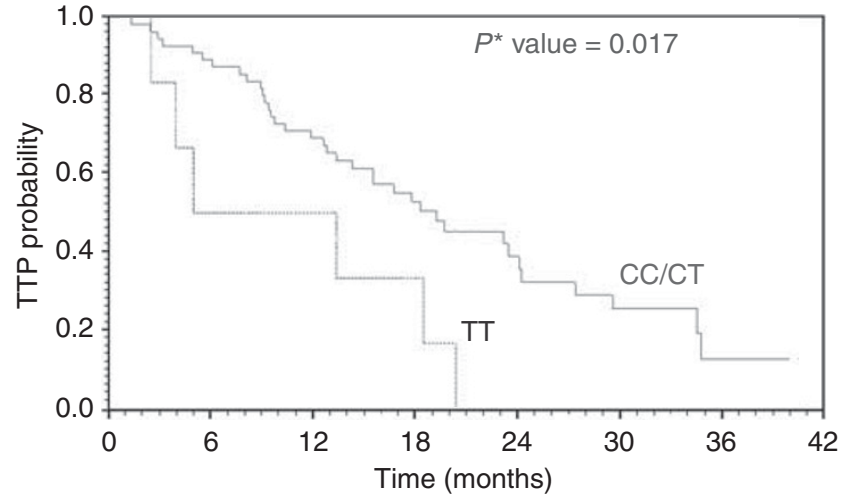

Figure 1 Kaplan-Meier method was used to assess the effect of singlenucleotide polymorphism (SNP) C > T rs7372209 in pri-miR-26a-1 on time to progression (TTP) in 61 patients. $P$-value was calculated using the log-rank test.

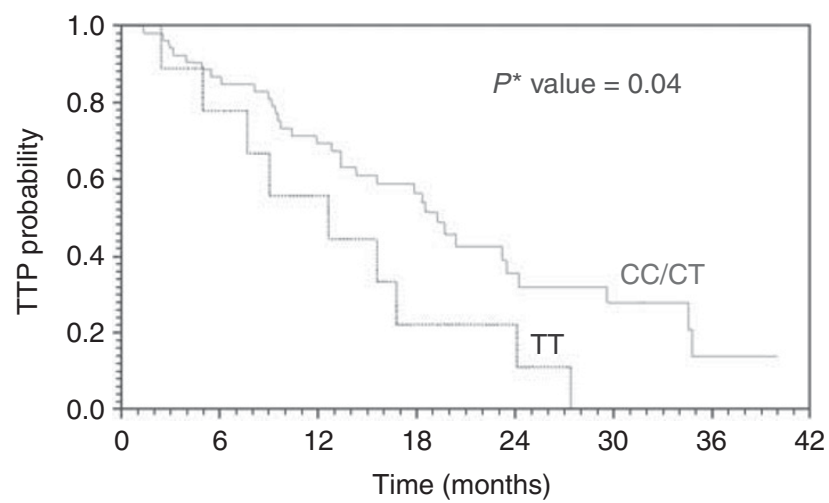

Figure 2 Kaplan-Meier method was used to assess the effect of singlenucleotide polymorphism (SNP) C $>T$ rs1834306 in pri-miR-100 gene on time to progression (TTP) in 61 patients. $P$-value was calculated using the log-rank test.

a statistically significant association with OS. As noted, primary tumor resection was associated with an OS benefit in univariate but not in multivariate analysis. 
Table 4 Putative target genes for miR-26a-1 and miR-100 predicted by TargetScan Human and miRANDA programs

Putative target genes References

miR-26a-1

SMAD1 (SMAD family member 1 )

TET1 (tet oncogene 1)

E2F7 (E2F transcription factor 7)

RB1 (retinoblastoma 1)

CCND2 (cyclin D2)

CCNE (cyclin E)

IGF1 (insulin-like growth factor 1)

$\mathrm{EZH} 2$ (enhancer of zeste homolog 2,

Drosophila)

PTEN (phosphatase and tensin homolog)

Kota et al. ${ }^{40}$

Sander et al. ${ }^{39}$

Huse et $a .^{41}$

$\operatorname{miR}-100$

CYP26B1 (cytochrome P450, family 26, subfamily $B$, polypeptide 1 )

HOXA1 (homeobox A1)

PLK1 (Polo-like kinase 1)

Shi et al. ${ }^{45}$

TargetScan Human (version 5.1) and miRANDA programs are available online at: http://www.targetscan.org/ and http://www.microrna.org/, respectively.

In silico analysis of miRNA putative targets

To explore the implications of our identified SNPs in translational regulation, we used the TargetScan Human (version 5.1$)^{34}$ and miRANDA ${ }^{35}$ programs. We identified a list of putative genes targeted by miR-26a-1 and miR-100 (summarized in Table 4). Moreover, we performed a PubMed search with the two miRNAs as the keywords, selecting articles in which putative target genes for these miRNAs were previously functionally validated. These references are given in Table 4.

\section{Discussion}

The identification of molecular markers that can predict treatment response is one of the most important aims in pharmacogenomics and cancer research. Currently, there is a growing interest in the study of the association between miRNA expression in tumors and chemo- and radiosensitivity, both with regards to predicting or modulating sensitivity. Indeed, different miRNAs have been found to predict sensitivity to anticancer treatment; for example, miR-30c, miR-130a, and miR-335 are downregulated in various chemoresistant cell lines, and hsa-Let-7g and hsa-miR-181b are strongly associated with response to the 5-FU-based antimetabolite S- $1 .^{36}$ These findings provide evidence that the variability in expression levels of miRNAs within the cell may influence chemotherapy sensitivity.

Polymorphisms present in miRNA-processing machinery, in pri- and in pre-miRNAs, could potentially influence expression levels of mature miRNAs, broadly affecting miRNA function. At the post-transcriptional level, miRNAs regulate specific genes involved in multiple pathways, including cell death, cell proliferation, and stress resistance. Moreover, miRNAs can influence drug response, targeting proteins involved in the metabolism and/or the mechanism of action of specific drugs. Thus, several authors have defined miRNA pharmacogenomics as the study of miRNAs and polymorphisms affecting miRNA function to predict drug behavior and to improve drug efficacy. In this context, we investigated the potential correlation between SNPs in miRNA-containing genomic regions and in miRNA-biogenesis machinery genes and the clinical outcome of mCRC patients treated with first-line 5-fluoruracil and CPT-11 combination.

To our knowledge, our data are the first to provide evidence that common SNPs in miRNA-related genes may have a role in predicting clinical outcome in mCRC patients treated with 5-FU and CPT-11. We found that SNPs in miRNA-containing genomic regions and in miRNAbiogenesis machinery genes may be useful predictors of 5-FU and CPT-11 efficacy in mCRC patients. The SNP rs7372209 in miR-26-a-1 was associated with both ORR and TTP. Allele $\mathrm{C}$ appears to be a favorable factor, as was confirmed comparing the median TTP of CC + CT genotypes to the homozygote variant genotype (TT). Furthermore, the SNP rs1834306 in the $5^{\prime}$ UTR region of pri-miR-100 and the SNP rs11077 in the XPO5 gene were also found to be associated with TTP and DCR, respectively.

The molecular mechanism by which these polymorphisms lead to improving the efficacy of a drug is not yet understood. Indeed, for only a few miRNAs are the biological functions only beginning to be more clearly understood. In our opinion, these SNPs may affect miRNA primary transcription and then expression of the mature miRNA. Finally, the ectopic expression of mature miRNAs may affect the translational suppression of their targets and consequently influence drug response. Although it is not possible at this time to further define the mechanism through which these SNPs affect response to therapy, several studies have reported a correlation between miRNA expression and the effect on sensitivity to chemotherapy. For example, the sequence variations in miRNA genes, including pri-miRNAs, pre-miRNAs, and mature miRNAs, could potentially influence the processing and/or target selection of miRNAs. ${ }^{6}$ These findings support our initial hypothesis that some variant alleles in microRNA-containing genome regions and/or in miRNA-processing machinery, by changing the expression of mature miRNAs, might be associated with cancer response. However, the elucidation of the mechanisms underlying SNP function and overcoming their role in chemotherapy resistance warrants further insight.

Interestingly, when we performed the in silico analysis for putative target genes of these miRNAs, we found that some of them have been implicated in colon cancer tumorigenesis and clinical outcome. For example, E2F transcription factor 7 (E2F7) and SMAD family member 1 (SMAD1) genes were identified by TargetScan as putative targets of miR-26a-1. E2F transcription factors, such as E2F7, have an essential role in the regulation of cell-cycle progression, ${ }^{37}$ and SMAD 
proteins are members of the transforming growth factor- $\beta$ pathway protein family, involved in regulation of cell proliferation, differentiation, and apoptosis. ${ }^{38}$ Furthermore, recent studies have shown that the ectopic expression of miR-26a influences cell-cycle progression by targeting the bona fide oncogene enhancer of zeste homolog 2 (EZH2), a polycomb protein, and global regulator of gene expression $^{39}$; in addition, a recent work has shown that in liver cancer cells, ectopic expression of miR-26a induces cell-cycle arrest by direct targeting of cyclins D2 and E2. ${ }^{40}$ Finally, Huse et al. ${ }^{41}$ reported that miR-26a is a direct regulator of the expression of phosphatase and tensin homolog, a tumor suppressor gene frequently altered in cancer. ${ }^{42}$

Interestingly, the putative target genes of miR-100 predicted by the TargetScan Human program may be involved in colon cancer pathogenesis, as well. For instance, we found that one potential target of miR-100 is the gene HomeoboxA1 (HOXA1). Furthermore, in searching using the miRANDA program, as putative target gene, insulin-like growth factor 1 receptor $(I G F 1 R)$ was found. This gene is highly overexpressed in most malignant tissues, where its protein functions as an anti-apoptotic agent by enhancing cell survival, and recent evidence suggests that $I G F 1 R$ is a key player in cancer therapeutic resistance. ${ }^{43}$ Recent reports have also shown that a decreased expression of miR-100 contributes to malignancy in oral cancer cells and that it is associated with loss of sensitivity to ionizing radiation. ${ }^{44}$ For the validated target of miR-100, we found only the Polo-like kinase 1 (Plk1) gene, a key regulator of mitosis. ${ }^{45}$ Furthermore, $P l k 1$ has been proposed as a new prognostic biomarker in colon cancer patients. ${ }^{46}$ Finally, the SNP rs11077 in the XPO5 gene showed a trend toward an association with DCR. The exportin-5 protein is a pre-miRNA-specific export carrier involved in shuttling pre-miRNA into the cytoplasm through a saturable carrier-mediated process. ${ }^{47}$ It is possible that SNPs in the $3^{\prime} \mathrm{UTR}$ region of XPO5 could be associated with altered expression levels of this protein and consequently with variations in mature miRNA expression in the cytoplasm.

Lastly, two SNPs, the rs3757 and the rs2368392, were found in Hardy-Weinberg disequilibrium in our population. We argued three possible explanations for these findings: first, it could be due to linkage disequilibrium with other near loci. Loci that are phenotypically neutral but are somehow associated with the disease-susceptibility locus, such as genetic markers in linkage disequilibrium with the disease-susceptibility locus, experience disproportionate genotype selection. Second, the small number of patients recruited in the study could explain these departures. Finally, these polymorphisms could be associated with colorectal cancer risk. Alleles within genotypes that confer greater susceptibilities are usually represented in the sample at disproportionally high rates. However, a case-control study would be necessary to investigate this last interesting hypothesis.

In conclusion, we investigated the influence of SNPs in miRNA-related genes on clinical outcome in mCRC patients homogeneously treated with 5-FU and CPT-11. Although our results must be validated in larger prospective studies, they highlight a potential role of these SNPs as predictive biomarkers. Changes in precursor and primary sequences of miRNAs could have an important effect on post-transcriptional regulation, influencing miRNA expression levels and consequently the cellular proteomic network. The described putative and validated targets of miR-26 and miR-100 are known as important genes involved in colon cancer development, although they have not directly been related to 5-FU and/or irinotecan metabolism or mechanism of action. We may therefore speculate that our relevant SNPs may be associated with response to treatment but not with a response to a specific drug combination. Unfortunately, the design of our study precludes further conclusions. Future studies are necessary to explore the functional role of this new class of SNPs. Finally, in the future, these SNPs may be used as a decision-making tool to assist oncologists in selecting the most appropriate systemic therapy for each patient.

\section{Conflict of interest}

The authors declare no conflict of interest.

\section{Acknowledgments}

We thank Massimiliano Copetti for helping with statistical analysis and Paola Parrella for careful comments on this manuscript. We also thank Ines Lopez Erdozain and Marisol González Huarriz for their excellent technical assistance.

\section{References}

1 Jemal A, Siegel R, Ward E, Hao Y, Xu J, Thun MJ. Cancer statistics, 2009. CA Cancer / Clin 2009; 59: 225-249.

2 Meng F, Henson R, Lang M, Wehbe H, Maheshwari S, Mendell JT et al. Involvement of human micro-RNA in growth and response to chemotherapy in human cholangiocarcinoma cell lines. Gastroenterology 2006; 130: 2113-2129.

3 Bandres E, Bitarte N, Arias F, Agorreta J, Fortes P, Agirre X et al. microRNA-451 regulates macrophage migration inhibitory factor production and proliferation of gastrointestinal cancer cells. Clin Cancer Res 2009; 15: 2281-2290.

4 Song B, Wang Y, Kudo K, Gavin EJ, Xi Y, Ju J. miR-192 Regulates dihydrofolate reductase and cellular proliferation through the p53-microRNA circuit. Clin Cancer Res 2008; 14: 8080-8086.

5 Calin GA, Dumitru CD, Shimizu M, Bichi R, Zupo S, Noch E et al. Frequent deletions and down-regulation of micro- RNA genes miR15 and miR1 6 at $13 q 14$ in chronic lymphocytic leukemia. Proc Natl Acad Sci USA 2002; 99: 15524-15529.

6 Duan R, Pak C, Jin P. Single nucleotide polymorphism associated with mature miR-125a alters the processing of pri-miRNA. Hum Mol Genet 2007; 16: 1124-1131.

7 Saunders MA, Liang $\mathrm{H}$, Li WH. Human polymorphism at microRNAs and microRNA target sites. Proc Natl Acad Sci USA 2007; 104: 3300-3305.

8 Iwai N, Naraba H. Polymorphisms in human pre-miRNAs. Biochem Biophys Res Commun 2005; 331: 1439-1444.

9 Mishra PJ, Bertino JR. MicroRNA polymorphisms: the future of pharmacogenomics, molecular epidemiology and individualized medicine. Pharmacogenomics 2009; 10: 399-416.

10 Passetti F, Ferreira CG, Costa FF. The impact of microRNAs and alternative splicing in pharmacogenomics. Pharmacogenomics / 2009; 9: $1-13$. 
11 Iorio MV, Ferracin M, Liu CG, Veronese A, Spizzo R, Sabbioni S et al MicroRNA gene expression deregulation in human breast cancer. Cancer Res 2005; 65: 7065-7070.

12 Lu J, Getz G, Miska EA, Alvarez-Saavedra E, Lamb J, Peck D et al. MicroRNA expression profiles classify human cancers. Nature 2005; 435: 834-838

13 Volinia S, Calin GA, Liu CG, Ambs S, Cimmino A, Petrocca F et al. A microRNA expression signature of human solid tumors defines cancer gene targets. Proc Natl Acad Sci USA 2006; 103: 2257-2261.

14 Calin GA, Croce CM. MicroRNA signatures in human cancers. Nat Rev Cancer 2006; 6: 857-866.

15 Zhang L, Coukos G. MicroRNAs: a new insight into cancer genome. Cell Cycle 2006; 5: 2216-2219.

16 He L, Thomson JM, Hemann MT, Hernando-Monge E, Mu D, Goodson $S$ et al. A microRNA polycistron as a potential human oncogene. Nature 2005; 435: 828-833.

17 Bandres E, Agirre X, Bitarte N, Ramirez N, Zarate R, Roman-Gomez et al. Epigenetic regulation of microRNA expression in colorectal cancer. Int J Cancer 2009; 125: 2737-2743.

18 Lee Y, Kim M, Han J, Yeom KH, Lee S, Baek SH et al. MicroRNA genes are transcribed by RNA polymerase II. EMBO J 2004; 23: 4051-4060.

19 Cullen BR. Transcription and processing of human microRNA precursors. Mol Cell 2004; 16: 861-865.

20 Lee Y, Ahn C, Han J, Choi H, Kim J, Yim J et al. The nuclear RNase III Drosha initiates microRNA processing. Nature 2003; 425: 415-419.

21 Yi R, Qin Y, Macara IG, Cullen BR. Exportin-5 mediates the nuclear export of pre-microRNAs and short hairpin RNAs. Genes Dev 2003; 17 3011-3016.

22 Chendrimada TP, Gregory RI, Kumaraswamy E, Norman J, Cooch N Nishikura $\mathrm{K}$ et al. TRBP recruits the Dicer complex to Ago2 for microRNA processing and gene silencing. Nature 2005; 436: 740-744.

23 Bartel DP. MicroRNAs: genomics, biogenesis, mechanism, and function. Cell 2004; 116: 281-297.

24 Mourelatos Z, Dostie J, Paushkin S, Sharma A, Charroux B, Abel L et al miRNPs: a novel class of ribonucleoproteins containing numerous microRNAs. Genes Dev 2002; 16: 720-728.

25 Hutvagner G, Zamore PD. A microRNA in a multiple-turnover RNA enzyme complex. Science 2002; 297: 2056-2060.

26 Tang G. siRNA and miRNA: an insight into RISCs. Trends Biochem Sci 2005; 30: 106-114.

27 Zeng Y, Yi R, Cullen BR. MicroRNAs and small interfering RNAs can inhibit mRNA expression by similar mechanisms. Proc Natl Acad Sci USA 2003; 100: 9779-9784.

28 Therasse P, Arbuck SG, Eisenhauer EA, Wanders J, Kaplan RS, Rubinstein $\mathrm{L}$ et al. New guidelines to evaluate the response to treatment in solid tumors. European Organization for Research and Treatment of Cancer, National Cancer Institute of the United States, National Cancer Institute of Canada. I Natl Cancer Inst 2000; 92: 205-216.

29 Poston G], Figueras I, Giuliante F, Nuzzo G, Sobrero AF, Gigot JF et al. Urgent need for a new staging system in advanced colorectal cancer. J Clin Oncol 2008; 26: 4828-4833.

30 Sherry ST, Ward MH, Kholodov M, Baker J, Phan L, Smigielski EM et al. dbSNP: the NCBI database of genetic variation. Nucleic Acids Res 2001 29: 308-311.

31 Horikawa $Y$, Wood CG, Yang $H$, Zhao $H$, Ye $Y$, Gu J et al. Single nucleotide polymorphisms of microRNA machinery genes modify the risk of renal cell carcinoma. Clin Cancer Res 2008; 14: 7956-7962.
32 Yang H, Dinney CP, Ye Y, Zhu Y, Grossman HB, Wu X. Evaluation of genetic variants in microRNA-related genes and risk of bladder cancer. Cancer Res 2008; 68: 2530-2537.

33 Westfall PH, Young SS. Resampling-Based Multiple Testing: Examples and Methods for P-Value Adjustment. John Wiley \& Sons, Inc: New York, 1993.

34 Lewis BP, Burge CB, Bartel DP. Conserved seed pairing, often flanked by adenosines, indicates that thousands of human genes are microRNA targets. Cell 2005; 120: 15-20.

35 John B, Enright AJ, Aravin A, Tuschl T, Sander C, Marks DS. Human MicroRNA targets. PLoS Biol 2004; 2: e363.

36 Hummel R, Hussey DJ, Haier J. MicroRNAs: predictors and modifiers of chemo- and radiotherapy in different tumour types. Eur / Cancer 2010; 46: 298-311.

37 Di Stefano L, Jensen MR, Helin K. E2F7, a novel E2F featuring DP-independent repression of a subset of E2F-regulated genes. EMBO J 2003; 22: 6289-6298.

38 Korchynskyi O, Landstrom M, Stoika R, Funa K, Heldin CH, ten Dijke P et al. Expression of Smad proteins in human colorectal cancer. Int / Cancer 1999; 82: 197-202.

39 Sander S, Bullinger L, Klapproth K, Fiedler K, Kestler HA, Barth TF et al. MYC stimulates $E Z H 2$ expression by repression of its negative regulator miR-26a. Blood 2008; 112: 4202-4212.

40 Kota J, Chivukula RR, O'Donnell KA, Wentzel EA, Montgomery CL, Hwang HW et al. Therapeutic microRNA delivery suppresses tumorigenesis in a murine liver cancer model. Cell 2009; 137: 1005-1017.

41 Huse JT, Brennan C, Hambardzumyan D, Wee B, Pena I, Rouhanifard SH et al. The PTEN-regulating microRNA miR-26a is amplified in high-grade glioma and facilitates gliomagenesis in vivo. Genes Dev 2009; 23: 1327-1337.

42 Wee $\mathrm{S}$, Jagani $\mathrm{Z}$, Xiang KX, Loo A, Dorsch M, Yao YM et al. PI3K pathway activation mediates resistance to MEK inhibitors in KRAS mutant cancers. Cancer Res 2009; 69: 4286-4293.

43 Casa AJ, Dearth RK, Litzenburger BC, Lee AV, Cui X. The type I insulinlike growth factor receptor pathway: a key player in cancer therapeutic resistance. Front Biosci 2008; 13: 3273-3287.

44 Henson BJ, Bhattacharjee S, O'Dee DM, Feingold E, Gollin SM. Decreased expression of miR-125b and miR-100 in oral cancer cells contributes to malignancy. Genes Chromosomes Cancer 2009; 48: 569-582.

45 Shi W, Alajez NM, Bastianutto C, Hui AB, Mocanu JD, Ito E et al. Significance of Plk1 regulation by miR-100 in human nasopharyngeal cancer. Int J Cancer 2009; 126: 2036-2048.

46 Weichert W, Kristiansen G, Schmidt M, Gekeler V, Noske A, Niesporek S et al. Polo-like kinase 1 expression is a prognostic factor in human colon cancer. World / Gastroenterol 2005; 11: 5644-5650.

$47 \mathrm{Yi} R$, Doehle BP, Qin $\mathrm{Y}$, Macara IG, Cullen BR. Overexpression of exportin 5 enhances RNA interference mediated by short hairpin RNAs and microRNAs. RNA 2005; 11: 220-226.

This work is licensed under the Creative Commons Attribution-NonCommercial-No Derivative Works 3.0 Unported License. To view a copy of this license, visit http://creativecommons.org/ licenses/by-nc-nd/3.0/

Supplementary Information accompanies the paper on the The Pharmacogenomics Journal website (http://www.nature.com/tpj) 